\title{
Voltage-dependent anion channel (VDAC) is involved in apoptosis of cell lines carrying the mitochondrial DNA mutation Liu Yuqi ${ }^{\dagger 1}$, Gao Lei ${ }^{\dagger 1}$, Li Yang ${ }^{\dagger 1}$, Li Zongbin ${ }^{1}, \mathrm{Xu} \mathrm{Hua}^{2}$, Wang Lin ${ }^{1}$, Chen Rui ${ }^{1}$, Liu Mohan ${ }^{1}$, Wen Yi ${ }^{1}$, Guan Minxin*3 and Wang Shiwen ${ }^{* 1}$
}

\author{
Address: ${ }^{1}$ Institute of Geriatric Cardiology, Chinese PLA General Hospital, Beijing, 100853, PR China, ${ }^{2}$ Military Medical Science Academy of the \\ Chinese PLA, Beijing, 100850, PR China and ${ }^{3}$ Cincinnati Children's Hospital Medical Center, Division of Human Genetics, Cincinnati, OH 45229, \\ USA \\ Email: Liu Yuqi - ametuofo4290@gmail.com; Gao Lei - guanmx@163.com; Li Yang - liyangbsh@163.com; Li Zongbin - lizww@yahoo.com.cn; \\ Xu Hua -xueh@gmail.com; Wang Lin - wanglin@gmail.com; Chen Rui - guanmx@163.com; Liu Mohan - qiaolaomao2@yahoo.com.cn; \\ Wen Yi - wenyi301@yahoo.com.cn; Guan Minxin* - guanmx@163.com; Wang Shiwen* - ametuofo980869@163.com \\ * Corresponding authors †Equal contributors
}

Published: 9 November 2009

BMC Medical Genetics 2009, I0:1/4 doi:10.1 186/1471-2350-10-1/4
Received: 12 October 2008

Accepted: 9 November 2009

This article is available from: http://www.biomedcentral.com/I47/-2350/I0/II4

(c) 2009 Yuqi et al; licensee BioMed Central Ltd.

This is an Open Access article distributed under the terms of the Creative Commons Attribution License (http://creativecommons.org/licenses/by/2.0), which permits unrestricted use, distribution, and reproduction in any medium, provided the original work is properly cited.

\begin{abstract}
Background: The mitochondrial voltage-dependent anion channel (VDAC) is increasingly implicated in the control of apoptosis. We have studied the effects the mitochondrial DNA (mtDNA) tRNAlle mutation on VDAC expression, localization, and apoptosis.

Methods: Lymphoblastoid cell lines were derived from 3 symptomatic and I asymptomatic members of a family with hypertension associated with the A4263G tRNAlle mutation as well as from control subjects. Mitochondrial potential $\left(\Delta \Psi_{\mathrm{m}}\right)$ and apoptosis were measured by flow cytometry; co-localization of VDAC and Bax was evaluated by confocal microscopy.
\end{abstract}

Results: Expression of VDAC and Bax in mtDNA cell lines was found to be increased compared to controls, while expression of the small conductance calcium-dependant potassium channel $\left(\mathrm{sK}_{\mathrm{C}_{\mathrm{a}}}\right)$ was unchanged. Confocal imaging revealed co-localization of VDAC/Bax on the outer mitochondrial membrane of A4263G cell lines but not from controls. Flow cytometry indicated that the mitochondrial potential was decreased by $32 \%$ in mutated cells versus controls while rates of apoptosis were increased $(P<0.05)$. The difference was attenuated by Cyclosporin $A(C s A, 2$ $\mu \mathrm{M})$, a blocker of VDAC.

Conclusion: We conclude that increased expression of mitochondrial VDAC and subcellular colocalization of VDAC/Bax increases mitochondrial permeability and apoptosis in cell lines carrying the mtDNA tRNAlle A4263G mutation.

\section{Background}

Hypertension is an established risk factor for coronary heart disease, stroke, congestive heart failure and renal dysfunction, and is the major modifiable risk factor of poor prognosis in a variety of cardiovascular diseases.
Multiple environmental and genetic factors are known to predispose to essential hypertension, with genetic predisposition contributing to $30-60 \%$ of the pathoetiology of the disease [1]. We previously reported on families with an inherited disposition to essential hypertension; some 
families showed an obvious pattern of maternal inheritance indicative of a mitochondrial disorder [2-5]. In a previous study on a large Chinese Han family with maternally-inherited hypertension we uncovered a mutation in the mitochondrial A4263G tRNAlle gene. The mutation was found to affect a nucleotide conserved from bacteria to human. Importantly, the change was inferred to influence amino acid charging of tRNA [6] and was therefore likely to lead to translational amino-acid substitutions in some mitochondrial proteins, with major consequences for mitochondrial function.

Recent studies have reported that the outer mitochondrial membrane voltage-dependent anion channel (VDAC) is associated with type 2 diabetes mellitus [7-9], an important finding in view of the link between diabetes and mitochondrial function $[10,11]$. There are 3 kinds of VDAC 1 to 3, but in human VDAC1 is highly expressed, which controls the transit of adenine nucleotides, $\mathrm{Ca}^{2+}$, and other metabolites $[12,13]$ both into and out of the mitochondrion. The channel is also a constituent of the mitochondrial permeability transition pore (PTP) [14-16] and therefore is likely to play a central role in the control of apoptosis. To study the link between the mitochondrial tRNA ${ }^{\text {Ile }}$ A4263G mutation, high blood pressure, and apoptosis we established lymphoblastoid cell lines from individuals carrying the A4263G mutation and from controls. We report that the A4263G mutation is associated with changes in VDAC expression, localization, and levels of apoptosis.

\section{Methods}

\section{Cell lines and culture conditions}

Informed consent, blood samples were obtained from all of the participating family members, under protocols approved by the ethics committee of Chinese PLA General Hospital and the Cincinnati Children's Hospital Medical Center Institute Review Board. Lymphoblastoid cell lines were immortalized by transformation with Epstein-Barr virus as described elsewhere [17]. Cell lines derived from 4 members of the Chinese family with a maternally-inherited predisposition to hypertension. Three individuals [II4, III-14, III-18] displayed clinical hypertension, one individual [III-19] was asymptomatic (see Figure 1). Cell lines were also isolated by the same method from 3 genetically unrelated control individuals (A1, A2, A3). Cells were grown in RPMI 1640 medium (Gibco) supplemented with $15 \%$ fetal bovine serum (FBS).

\section{Expression of VDAC, Bax and $s K_{C a}$}

Cells lines carrying the mtDNA tRNA ${ }^{\text {Ile }} 4263 \mathrm{~A} \rightarrow \mathrm{G}$ and control cell lines were washed with ice-cold PBS and total RNA was isolated using the TRIzol reagent (Invitrogen) according to the manufacturer's instructions. RNA $(2 \mu \mathrm{g})$ was treated with ribonuclease-free deoxyribonuclease and cDNA was synthesized using Moloney murine leukemia virus reverse transcriptase (Invitrogen Life Technologies, Carlsbad, CA); cDNA $(2 \mu \mathrm{l})$ was subjected to 44 cycles of PCR amplification, generating a single specific amplification product of the expected size. PCR conditions were as follows: denaturation for $30 \mathrm{sec}$ at $94^{\circ} \mathrm{C}$, annealing for 1

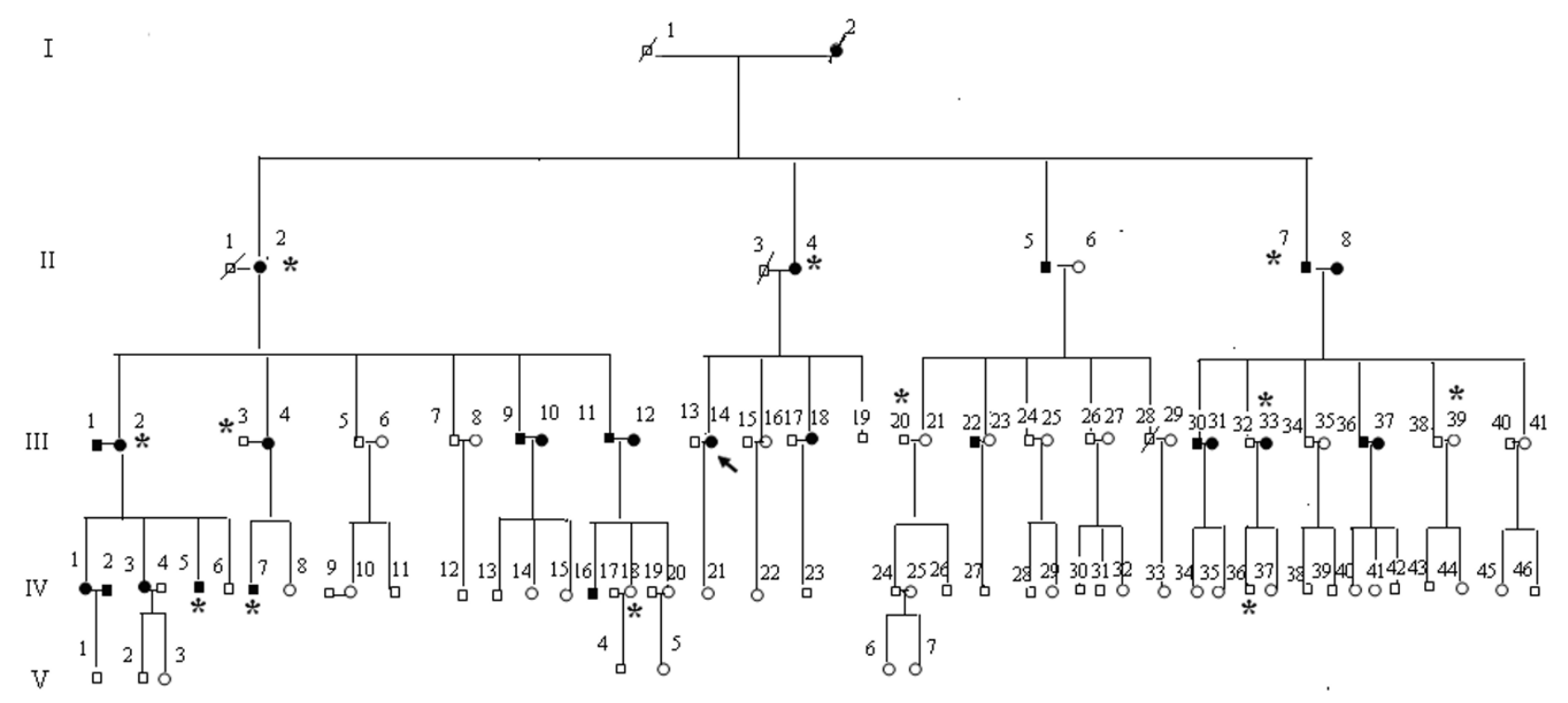

Figure I

The Han Chinese pedigree with a maternal pattern of inheritance of hypertension. Affected individuals are indicated by filled symbols. The arrowhead denotes the proband. * denotes cardiac hypertrophy. 
$\min$ at $55^{\circ} \mathrm{C}(\mathrm{VDAC}, \mathrm{Bax})$ or $58^{\circ} \mathrm{C}\left(\mathrm{sK}_{\mathrm{Ca}}\right)$, and $45 \mathrm{sec}$ extension at $72^{\circ} \mathrm{C}$. PCR primers used in this study were: VDAC sense, 5'-CTG AGT ACG GCC TGA CGT TT-3'; antisense 5'-ACT CTG TCC CGT CAT TCA CA-3'; Bax sense 5'GCA GCT TAA CGC ACC AAT TA-3', antisense 5'-CAG TTG AAG TTG CCG TCA GA-3'; sK $\mathrm{Ca}_{\mathrm{a}}$ sense 5'-GCA GCT TAA CGC ACC AAT TA-3', antisense 5'-TGA GGG AAA GGA CCA CTG AT-3'. Glyceraldehyde 3-phosphate dehydrogenase (GAPDH) was used as the PCR internal control using primers 5'-CTG CAC CAC CAA CTG CTT AG-3' (sense) and 5'-TTC AGC TCA GGG ATG ACC TT-3' (antisense). PCR reactions were in the linear range and were performed in triplicate; products were visualized by electrophoresis on $1.5 \%$ agarose gels and stained with ethidium bromide. Band intensities were normalized to GAPDH amplified in parallel and means were calculated from the triplicate reactions.

For the study of Cyclosporin A (CsA, an inhibitor of VDAC) on expression levels of VDAC, Bax and $\mathrm{sK}_{\mathrm{Ca}^{\prime}}$ cell lines were incubated in the presence of CsA (Novartis, 2 $\mu \mathrm{M}$ ) for $16 \mathrm{~h}$ before further analysis.

\section{Confocal microscopy}

Dual immunostaining was used to asssess the co-localization of Bax and VDAC-1 polypeptides. Cells were grown on glass coverslips in 6-well plates. After fixation (4\% paraformaldehyde in PBS), specimens were blocked with 5\% BSA for $15 \mathrm{~min}$, and incubated overnight with $200 \mu \mathrm{l}$ of primary antibody (anti-VDAC polyclonal antibody; Cell Signaling Technology, Danvers, MA; or anti-Bax polyclonal Antibody, NeoMarkers, Fremont, CA) diluted 1:100, followed by FITC green or rhodamine 123 labelled secondary antibody for $60 \mathrm{~min}$ at $37^{\circ} \mathrm{C}$, respectively. After washing, slides were mounted with cover slips and imaged using a confocal laser scanning system (RADIANCE 2100, Bio-Rad, Hercules, CA). Excitation-emission used an Argon $488 \mathrm{~nm}$ laser in conjunction with a 505$525 \mathrm{~nm}$ filter for the Alexa Fluor 488, and a HeNe 543 laser with a $610 \mathrm{~nm}$ filter for the Alexa Fluor 546 [18].

\section{Mitochondrial membrane potential and apoptosis measurements}

The mitochondrial membrane potential $\left(\Delta \Psi_{\mathrm{m}}\right)$ was monitored using the fluorescent reporter probe JC-1. Lymphoblastoid cell lines were incubated with $0.1 \mu \mathrm{M}$ JC-1 (Alexis; Portland, OR) for $10 \mathrm{~min}$ at $37^{\circ} \mathrm{C} \mathrm{[19].} \mathrm{After} \mathrm{this}$ loading period the cells were rinsed with phosphate-buffered saline (PBS)/bovine serum albumin and resuspended into $0.1 \mu \mathrm{M}$ JC- 1 in PBS/bovine serum albumin at room temperature [20]. $\Delta \Psi_{\mathrm{m}}$ was measured by cytofluorimetry (FL3). To evaluate the effects of CsA on $\Delta \Psi_{\mathrm{m}^{\prime}}$ cell lines were pre-incubated with CsA (Novartis, $2 \mu \mathrm{M}$ ) for 30 min and washed $5 \times$ in PBS before measurements. For determinations of levels of apoptosis, cells were stained with Annexin V/FITC and PI stain and imaged using laserscanning confocal microscopy (LSCM).

\section{Statistical analyses}

All data were presented as $\overline{\mathrm{X}} \pm \mathrm{SD}$. Comparison of continuous variables was performed using the unpaired Student's $t$ test. Statistical significance was set at $P<0.05$. Statistical analysis used SPSS software (version 11.0; SPSS Inc, Chicago, IL).

\section{Results}

Expression of VDAC, Bax and $s K_{C a}$

To study the effects of the mitochondrial tRNA ${ }^{\text {Ile }}$ A4263G mutation on mitochondrial function, lymphoblastoid cell lines were isolated from familial carriers of the mutation (Figure 1) and from controls. Quantitative RT-PCR was used to measure the mRNA expression levels of VDAC, Bax and $\mathrm{sK}_{\mathrm{Ca}}$. As shown in Figure 2, the A4263G mutation was associated with a significant $(P<0.05)$ increase in the levels of expression of both VDAC and Bax. There was no change in the expression level of a control channel, $\mathrm{sK}_{\mathrm{Ca}}$.

We then investigated the effects of Cyclosporin A (CsA), a selective inhibitor of VDAC, on VDAC and Bax mRNA levels. After incubation with CsA for $16 \mathrm{~h}$, VDAC mRNA levels decreased significantly and even lower than the control levels $(P<0.05)$ while there was no significant change in the levels of either Bax or $\mathrm{sK}_{\mathrm{Ca}}$ mRNA (Figure 2).

\section{Co-localization of VDAC and Bax protein}

VDAC was localized on the outer membrane of the mitochondrial, but Bax was expressed in the cytoplasm, transferred to outer membrane of mitochondrial and combined to VDAC under pathological state. To address the relative localizations of VDAC and Bax in cell lines carrying the mtDNA tRNA ${ }^{\text {Ile }}$ A4263G mutation, specific antibodies against these proteins were used for dual immunofluorescence on mutant cells and controls. Localization was imaged under confocal microscopy. Representative sections are shown in Figure 3.

Separate VDAC (green) and Bax (red) fluorescence was detected in control cell lines, indicating that the respective polypeptides were separately localized. In contrast, in cell lines from 2 tRNA Ile A4263G subjects (III-14 and III-19) co-localization of VDAC and Bax was revealed by intense yellow fluorescence.

We also investigated the effects of CsA on co-localization. Cell lines were incubated with CsA for $30 \mathrm{~min}$ prior to analysis. Confocal imaging revealed that CsA treatment abolished co-localization of VDAC and Bax. 
A

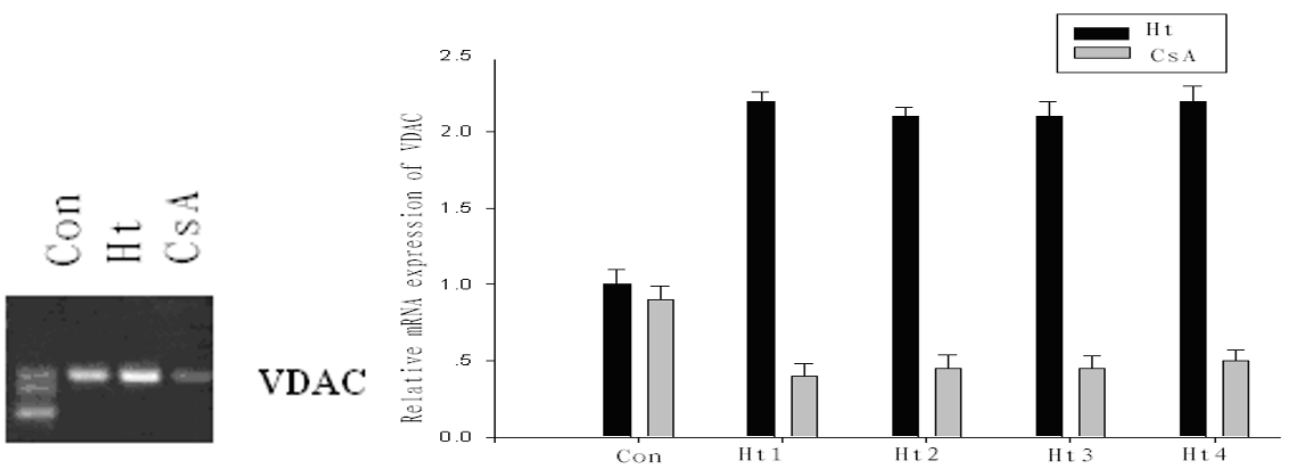

$\Xi \equiv \underset{3}{0}$

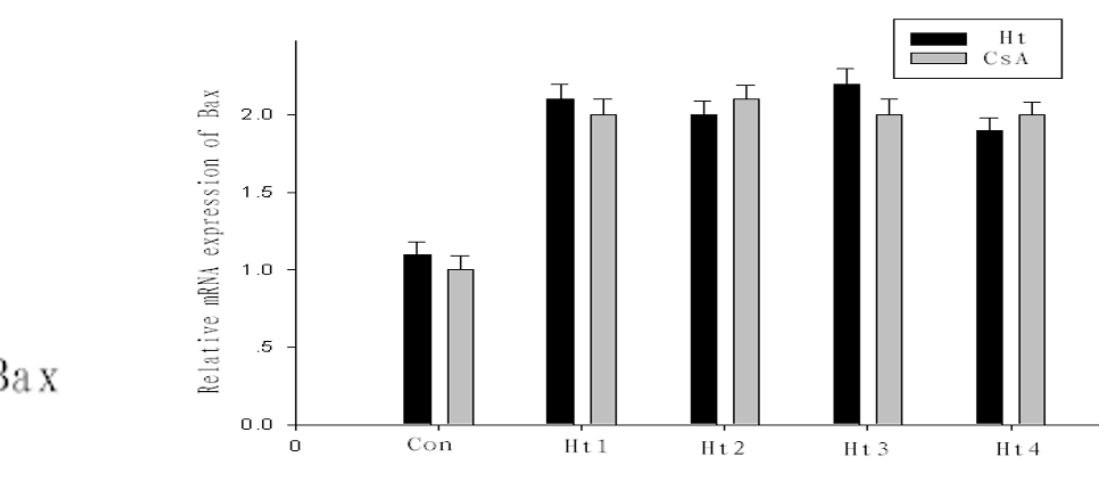

B
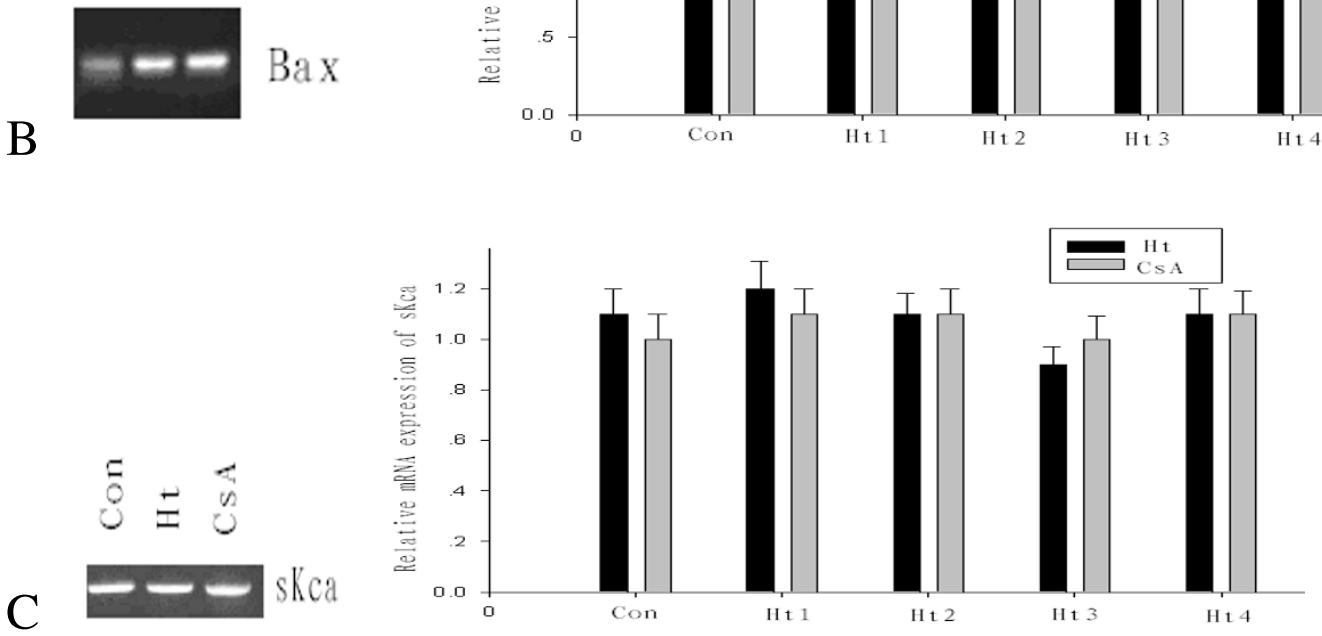

Figure 2

Expression of VDAC, Bax and $\mathbf{s} \mathbf{K}_{\mathbf{C a}_{\mathbf{a}}} \mathbf{m R N A s .}$ Con, cell line from the control group; $\mathrm{Ht}_{1}$, cell line from III- I4; $\mathrm{Ht}_{2}$, cell line from II-4; $\mathrm{Ht}_{3}$, cell line from III- I8; $\mathrm{Ht}_{4}$, cell line from III- 19; CsA, cell line from III-I4 after incubation with CsA (2 $\left.\mu \mathrm{M}\right)$ for I6 h. $A$, expression of VDAC mRNA; $B$, expression of Bax mRNA; C, expression of $s K_{C_{a}}$ channel mRNA.

Mitochondrial membrane potential $\left(\Delta \Psi_{m}\right)$ and apoptosis The magnitude of $\Delta \Psi_{\mathrm{m}}$ is controlled by the activity of the mitochondrial $\mathrm{K}_{\mathrm{ATP}}\left(\right.$ mito $\left._{\mathrm{ATP}}\right)$ and mito $_{\mathrm{Ca}}$ channels, as well as by the mitochondrial permeability transition pore (PTP) [21]. Cytometry images of lymphocytes loaded with JC-1, a specific reporter of $\Delta \Psi_{\mathrm{m}^{\prime}}$, revealed that membrane potential in tRNA ${ }^{\text {Ile }} \mathrm{A} 4263 \mathrm{G}$ cells was decreased by $32 \%$ compared to controls $(P<0.05)$. After incubation with CsA for $30 \mathrm{~min}$ the $\Delta \Psi_{\mathrm{m}}$ of both control and the mutated cell lines was increased; the increase was $33.6 \%$ in control cells but was $84.4 \%$ in III-14 mutant cells and $137.7 \%$ in III-19 mutant cells (Figure 4).

We then compared $\Delta \Psi_{\mathrm{m}}$ values between cell lines from controls and subjects carrying the A4263G mutation, both prior to and following incubation with CsA. As shown in Figure 5, $\Delta \Psi_{\mathrm{m}}$ values were significantly decreased $(P<$ 0.05 ) in both cell lines harboring the mutation. CsA pretreatment increased $\Delta \Psi_{\mathrm{m}}$ in all cell lines, and after CsA treatment there was no significant difference between $\Delta \Psi_{\mathrm{m}}$ values in control and mutant cells (Figure 5). 


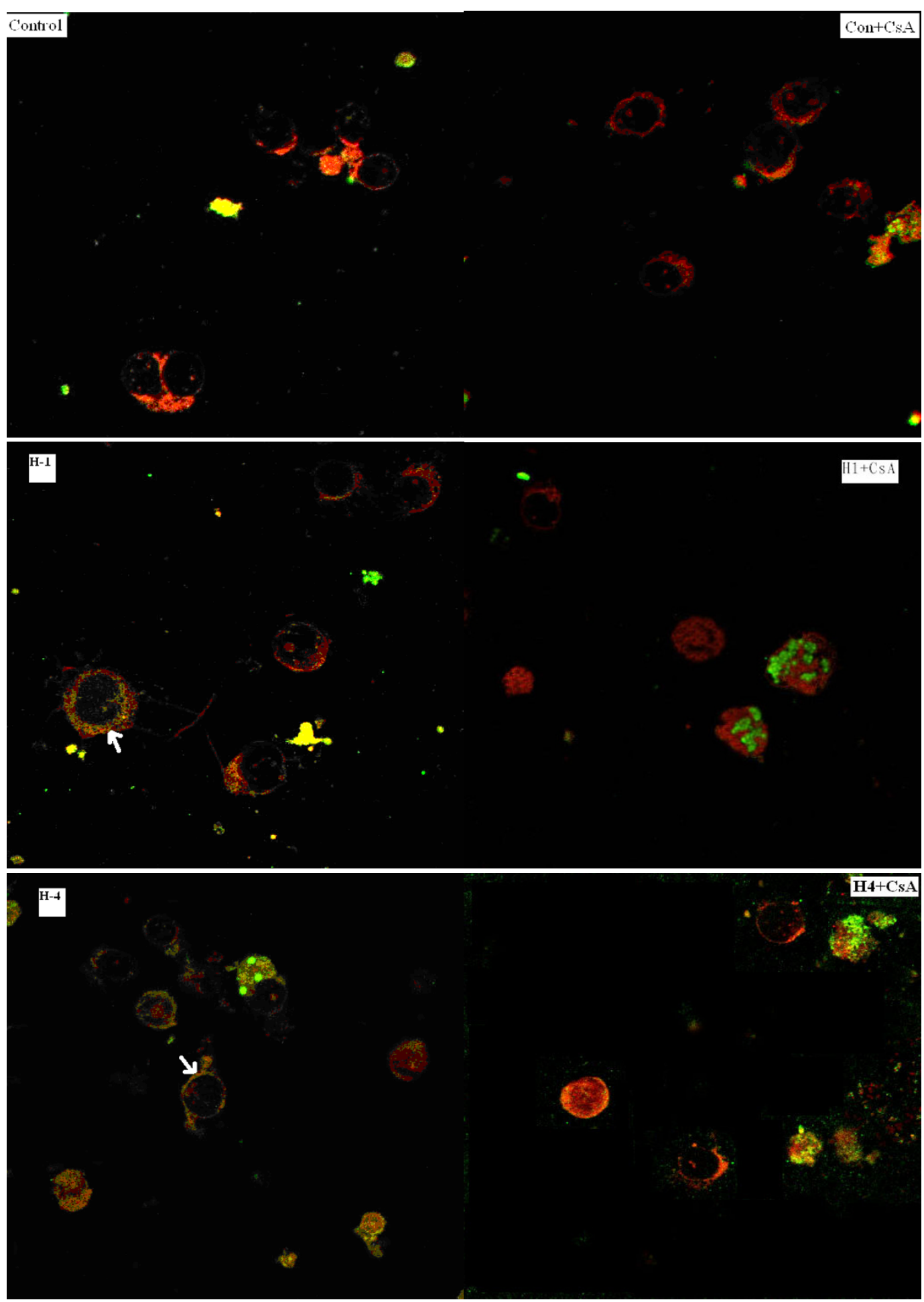

\section{Figure 3}

Localization of mitochondrial VDAC and Bax by confocal microscopy. Control, cell line from control group; Con+CsA, cell line incubated with CsA $(2 \mu \mathrm{M})$ for 30 min; $\mathrm{H}-\mathrm{I}$, cell line from III- $14 ; \mathrm{H}-\mathrm{I}+\mathrm{CsA}$, cell line incubated with CsA (2 $\mu \mathrm{M})$ for $30 \mathrm{~min}$; H-4, cell line from III- 19; H-4+CsA, cell line incubated with CsA (2 $\mu \mathrm{M})$ for 30 min. VDAC was stained with FITC-green (green fluorescence); Bax was stained with rhodamine 123 (red fluorescence). 
To determine whether changes in VDAC mRNA levels, localization, and $\Delta \Psi_{\mathrm{m}}$ values in lines from subjects carrying the A4263G mutation are associated with changes in apoptosis, cells were analyzed using Annexin V-FITC and flow cytometry, a sensitive assay for apoptosis. Levels of apoptosis were increased by $30 \%$ in cell lines carrying the mutation (Figure 6, left panel). However, the difference from controls was largely abolished by treatment with CsA; this reduced levels of apoptosis by $24.6 \%$ in control cells but by $56.9 \%$ in III-14 cells and by $67.1 \%$ in III-19 cells (Figure 6, right panel).

\section{Discussion}

Hypertension is a major risk factor for cardiovascular disease. Approximately 1 billion individuals worldwide and 130 million in China suffer from hypertension, and the rates of morbidity and mortality associated with essential hypertension (EHT) continue to rise [22]. Epidemiological studies have indicated that the genetic variance underlying the predisposition to EHT ranges from 30-60\% [1]. Mitochondrial DNA (mtDNA) mutations characterized by maternal inheritance may make a significant contribution [23-26]. The Framingham heart study [27] on blood pressure in 6421 participants from 1593 families estimated that the heritability due to maternal effects was 5\% for multivariable-adjusted long-term average systolic blood pressure, while the heritability of diastolic blood pressure due to maternal effects was $4 \%$.

We previously reported on a large Chinese Han family with a predisposition to hypertension that demonstrated a typical maternal pattern of inheritance. mtDNA sequence analysis revealed a A4263G mutation in the

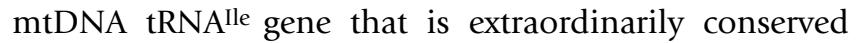
from bacteria to human. The voltage-dependent anion channel (VDAC) is a highly conserved protein located on the outer mitochondrial membrane [11]. VDAC, in association with ANT (adenine nucleotide translocator), mediates the transport of ATP and ADP both into and out of the mitochondrion [11]. VDAC closure inhibits the release of ATP from the mitochondrion and promotes the opening of $\mathrm{K}_{\mathrm{ATP}}$ channels in the plasma membrane. VDAC also mediates mitochondrial $\mathrm{Ca}^{2+}$ and may play a key role in intracellular $\mathrm{Ca}^{2+}$ signaling [28]. $t \mathrm{Bid}$, a pro-apoptotic member of the $\mathrm{Bcl}_{2}$ family, closes VDAC, and this may partly account for the inhibition of VDAC reported during apoptosis [29]. In contrast, anti-apoptotic Bcl-XL prevents VDAC closure, a finding consistent with the interpretation that VDAC opening is anti-apoptotic [30]. VDAC also appears to be an anchor point for pro- and anti-apoptotic proteins; it has been hypothesized that VDAC contributes to increase of mitochondrial permeability that is involved in the initiation of apoptosis.
We studied the effects of the mtDNA tRNA Ile A4263G mutation on VDAC function and apoptosis. We report that expression level of VDAC mRNA in cell lines carrying the mutation was significantly decreased. Although the exact mechanism is unknown, it may be associated with dysfunction of energy metabolism and consequent increase in the levels of reactive oxygen species (ROS) [31]. We hypothesis that high apoptosis of the mutated cell lines was associated with increased expression of VDAC mRNA, which could be inhibited by the CsA, a kind of inhibitor of VDAC. In addition, imaging revealed that VDAC was co-localized with Bax protein in cell lines carrying the $4263 \mathrm{~A} \rightarrow \mathrm{G}$ mutation. These changes were accompanied by a decrease in the mitochondrial membrane potential $\Delta \Psi_{\mathrm{m}}$ and increased levels of apoptosis versus control cell lines. The role of VDAC in these processes was confirmed by application of the selective VDAC inhibitor Cyclosporin A (CsA): the inhibitor abolished colocalization with Bax, restored $\Delta \Psi_{\mathrm{m}}$ to levels of control CsA-treated cells, and decreased levels of apoptosis. Taken together, these results suggest that the mtDNA A4263G mutation, inferred to cause mis-charging of tRNAlle and consequent amino acid substitutions in mitochondrial proteins, may exert its pro-hypertensive effects by deregulating the expression of VDAC, in turn leading to increases in programmed cell death.

So we conclude that increased expression of mitochondrial VDAC and subcellular co-localization of VDAC/Bax increases mitochondrial permeability and apoptosis in cell lines carrying the mtDNA tRNAlle A4263G mutation.

Recent efforts to identify genes involved in essential hypertension (EHT) have focused on genetic markers and candidate genes in the nuclear genome. Nevertheless, the pathophysiological mechanisms underlying EHT remain unknown. Our results highlight the potential importance of mitochondrial genes in the etiology of hypertension. However, this work does not exclude a contribution from the nuclear genome, and maternal inheritance of a predisposition to EHT is likely to result from interactions between mtDNA and nuclear mutations. So further research will be required to elucidate the mechanisms whereby nuclear-mitochondrial interactions can predispose to the development of hypertension.

Finally this article we conclude that increased expression of mitochondrial VDAC and subcellular co-localization of VDAC/Bax increases mitochondrial permeability and apoptosis.

\section{Competing interests}

The authors declare that they have no competing interests. 

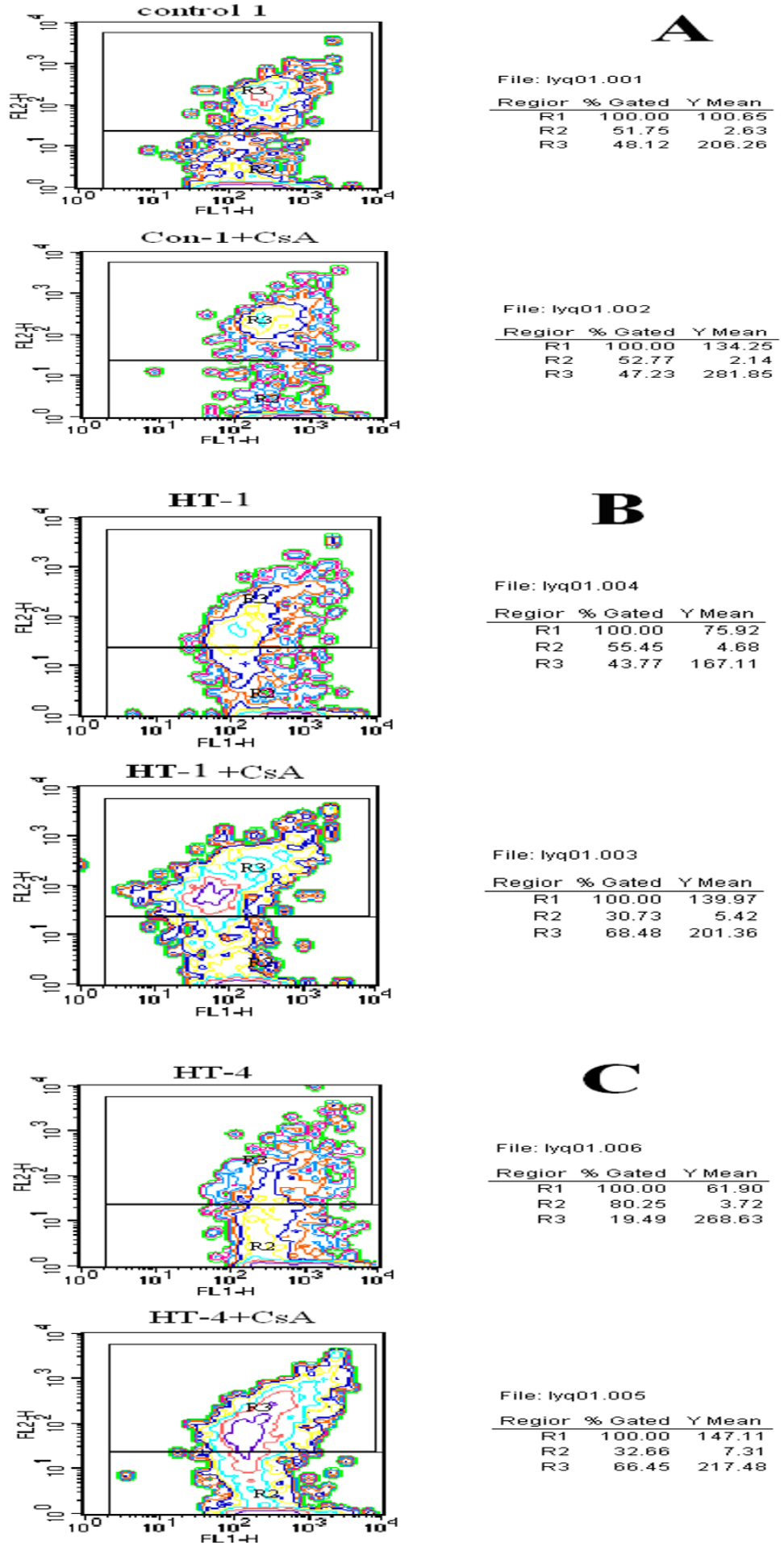
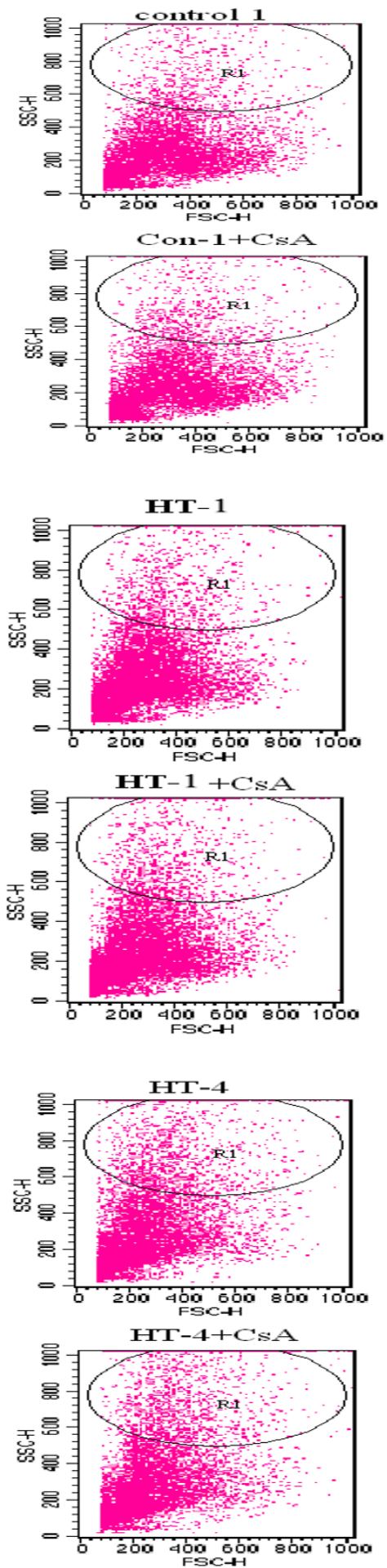

Figure 4

Mitochondrial potentials $\left(\Delta \Psi_{m}\right)$ of cell lines from III-I4, III-I 9 and from controls analyzed by flow cytometry. The left and right figures show $\Delta \Psi_{m}$ detected by flow cytometry before and after incubation with CsA (2 $\left.\mu M\right)$ for 30 min, respectively. A, cell lines of the control group; B, III-I4; C, III-I 9. 


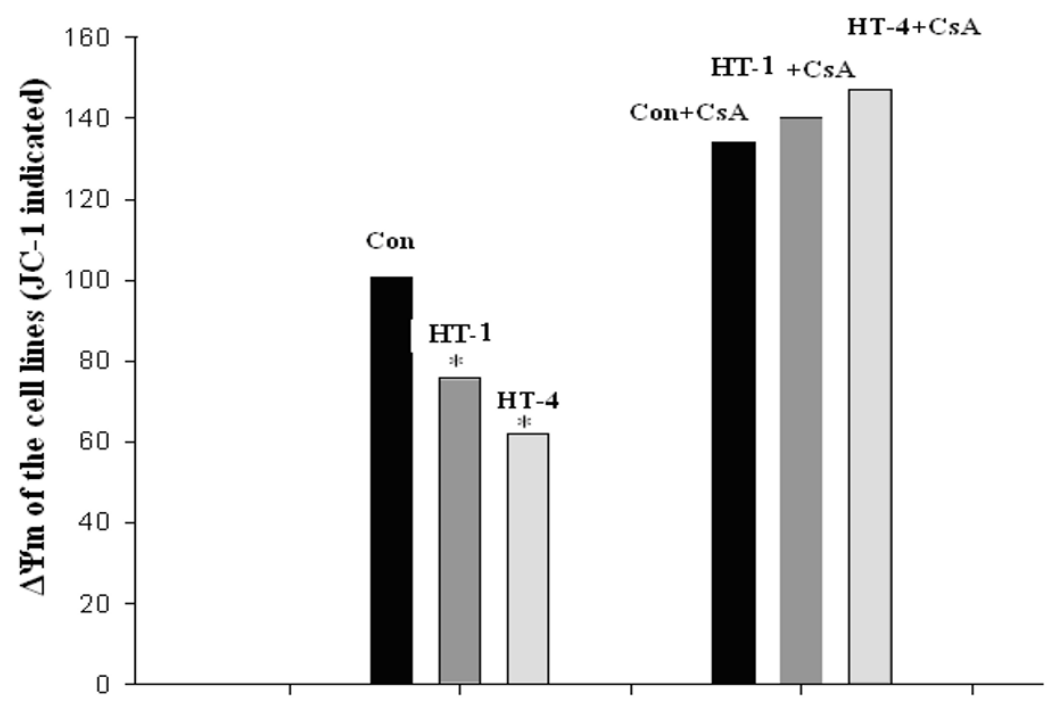

\section{Figure 5}

Comparison of $\Delta \Psi_{m}$ of cell lines from patients and controls. The average $\Delta \Psi_{m}$ value of cell lines from patients harboring the tRNA ${ }^{\text {lle }}$ A4263G mutation was decreased by $32 \%$ compared to control cell lines. $\Delta \Psi_{m}$ values of cell lines from both controls and patients were improved by $\mathrm{CsA}$; control values increased by $33.6 \%$, III-I 4 values increased by $84.4 \%$, and III- 19 values increased by $137.7 \%$.
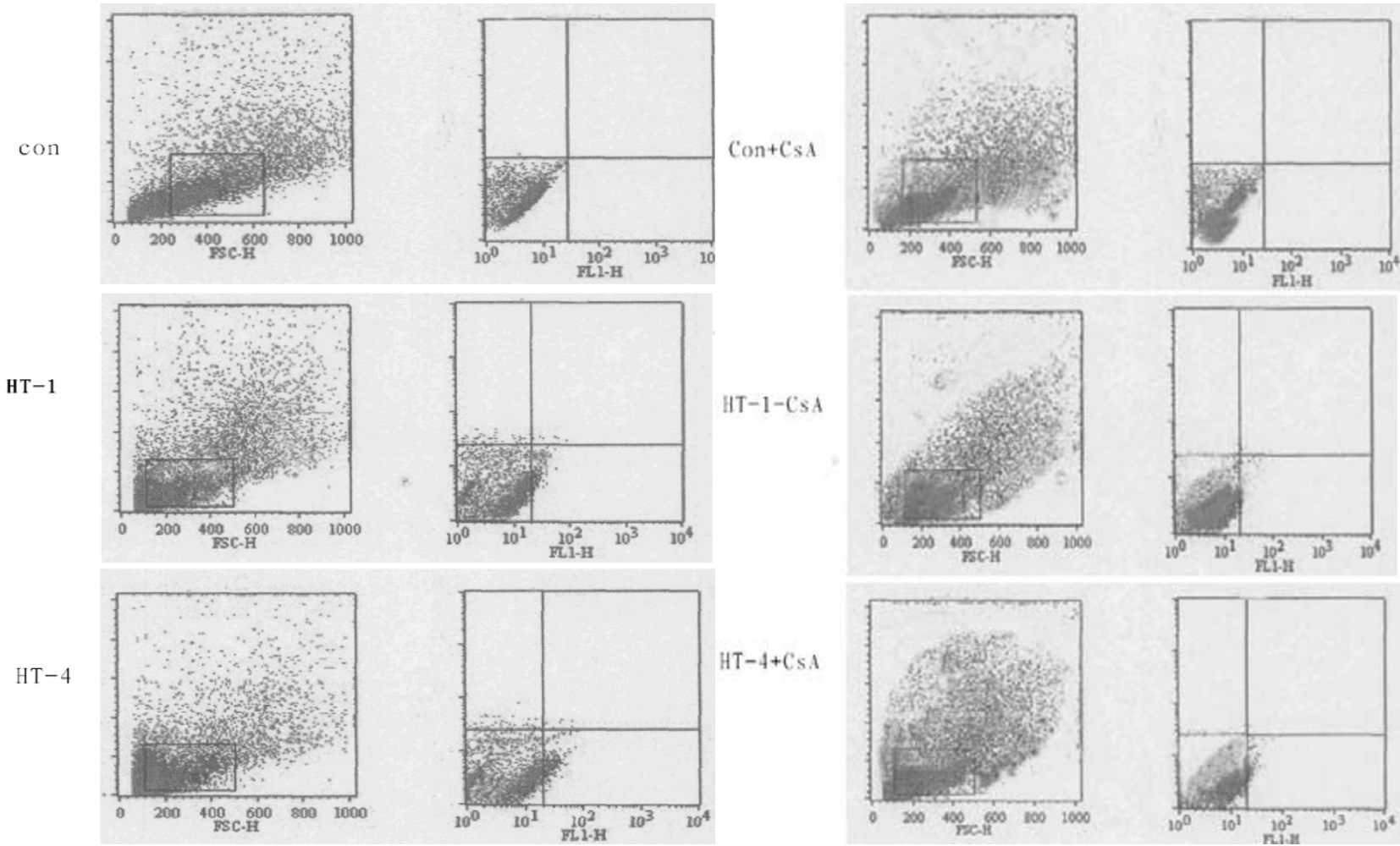

Figure 6

Apoptosis of cell lines from III-I4, III-I9, and controls. The left and right panels show flow cytometric analysis of apoptosis before and after incubation with CsA $(2 \mu \mathrm{M})$ for $30 \mathrm{~min}$, respectively. 


\section{Authors' contributions}

Liu Yuqi, Gao Lei, Li Yang contributed equally to this article. Li Zongbin carried out the molecular genetic studies, participated in the sequence analysis and drafted the manuscript. Xu Hua and Wang Lin carried out the immunoassays. Chen Rui, Liu Mohan and Wen Yi participated in the cell line culture. Wang Shiwen and Guan Minxin conceived of the study, and participated in its design and coordination. All authors read and approved the final manuscript.

\section{Acknowledgements}

This work was supported by National Institutes of Health (NIH) Grants ROIDC05230 and ROIDC07696 from the National Institute on Deafness and Other Communication Disorders to MXG and National Key Basic Research and Development Project 973 Fund 2007CB07403 to S.W.

\section{References}

I. Mein CA, Caulfield MJ, Dobson RJ, Munroe PB: Genetics of essential hypertension. Hum Mol Genet 2004, I 3:R I69-RI75.

2. Chung AB, Doyle JP, Wallace DC, Hall WD: The maternal inheritance of hypertension among African Americans. Am J Hypertens 1999, I 2:5A.

3. DeStefano AL, Gavras H, Heard-Costa N, Bursztyn M, Manolis A, Farrer LA, Baldwin CT, Gavras I, Schwartz F: Maternal component in the familial aggregation of hypertension. Clin Genet 200I, 60:13-2|.

4. Fuentes RM, Notkola IL, Shemeikka S, Tuomilehto J, Nissinen A: Familial aggregation of blood pressure: a population-based family study in eastern Finland. J Hum Hypertens 2000, 14:44I-445.

5. Sun F, Cui J, Gavras H, Schwartz F: A novel class of tests for the detection of mitochondrial DNA-mutation involvement in diseases. Am J Hum Genet 2003, 72: I5I5-I526.

6. Neefs JM, Peer Y Van de, De Rijik P, Goris A, De Wachter R: Compilation of small ribosomal subunit RNA sequences. Nucleic Acids Res 1991, 19:1987-2015.

7. Turko IV, Murad F: Quantitative Protein Profiling in Heart Mitochondria from Diabetic Rats. J Biol Chem 2003, 278:35844-35849.

8. Mostyn A, Pearce S, Stephenson T, Symonds ME: Hormonal and nutritional regulation of adipose tissue mitochondrial development and function in the newborn. Exp Clin Endocrinol Diabetes 2004, I I 2:2-9.

9. McCabe ER: Microcompartmentation of energy metabolism at the outer mitochondrial membrane: role in diabetes mellitus and other diseases. I Bioenerg Biomembr 1994, 26:3 17-325.

10. Shoshan-Barmatz $V$, Israelson $A$, Brdiczka D, Sheu SS: The voltagedependent anion channel (VDAC): function in intracellular signalling, cell life and cell death. Curr Pharm Des 2006, I 2:2249-2270.

II. Lemasters J], Holmuhamedov E: Voltage-dependent anion channel (VDAC) as mitochondrial governator - thinking outside the box. Biochim Biophys Acta 2006, I 762:18I-190.

12. Báthori G, Csordás G, Garcia-Perez C, Davies E, Hajnóczky G: Ca2+dependent control of the permeability properties of the mitochondrial outer membrane and voltage-dependent anion-selective channel (VDAC). J Biol Chem 2006, 28I:17347-17358.

13. Madesh M, Hajnóczky G: VDAC-dependent permeabilization of the outer mitochondrial membrane by superoxide induces rapid and massive cytochrome c release. J Cell Biol 200I, 155:1003-1015.

14. Szabó I, Zoratti M: The mitochondrial permeability transition pore may comprise VDAC molecules. I. Binary structure and voltage dependence of the pore. FEBS Lett 1993, 330:201-205.

15. Szabó I, De Pinto V, Zoratti M: The mitochondrial permeability transition pore may comprise VDAC molecules. II. The electrophysiological properties of VDAC are compatible with those of the mitochondrial megachannel. FEBS Lett 1993, 330:206-210.

16. Gincel D, Shoshan-Barmatz V: Glutamate interacts with VDAC and modulates opening of the mitochondrial permeability transition pore. J Bioenerg Biomembr 2004, 36: I79- 186.

17. Miller G, Lipman M: Release of infectious Epstein-Barr virus by transformed marmoset leukocytes. Proc Natl Acad Sci USA 1973, 70:190-194.

18. Yuste VJ, Sanchez-Lopez I, Sole C, Moubarak RS, Bayascas JR, Dolcet $X$, Encinas M, Susin SA, Comella JX: The contribution of apoptosis inducing factor, caspase activated DNase and inhibitor of caspase activated Dnase to the nuclear phenotype and degradation during apoptosis. $\mathrm{B}$ Biol Chem 2005, 280:5670-5683.

19. Mascarell L, Auger R, Alcover A, Ojcius DM, Jungas T, Cadet-Daniel V, Kanellopoulos JM, Truffa-Bachi P: Characterization of a Gene Encoding Two Isoforms of a Mitochondrial Protein Up-regulated by Cyclosporin A in Activated T Cells. J Biol Chem 2004, 279: 10556-10563.

20. Trollinger DR, Cascio WE, Lemasters JJ: Mitochondrial calcium transients in adult rabbit cardiac myocytes: inhibition by ruthenium red and artifacts caused by lysosomal loading of $\mathrm{Ca}^{2+}$-indicating fluorophores. Biophys J 2000, 79:39-50.

21. Sato T, Saito T, Saegusa N, Nakaya H: Mitochondrial $\mathbf{C a}^{2+}$-activated $\mathrm{K}^{+}$channels in cardiac myocytes: a mechanism of the cardioprotective effect and modulation by protein kinase $A$. Circulation 2005, I I I: 198-203.

22. Gu D, Reynolds K, Wu X, Chen J, Duan X, Muntner P, Huang G, Reynolds RF, Su S, Whelton PK, He J: Prevalence, awareness, treatment, and control of hypertension in china. Hypertension 2002, 40:920-927.

23. Brandao AP, Brandao AA, Araujo EM, Oliveira RC: Familial aggregation of arterial blood pressure and possible genetic influence. Hypertension 1992, 19:11214-11217.

24. Wallace DC: Mitochondrial defects in cardiomyopathy and neuromuscular disease. Am Heart J 2000, 139:S70-S85.

25. Hirano M, Davidson M, DiMauro S: Mitochondria and the heart. Curr Opin Cardiol 200I, 16:20I-2I0.

26. Schwartz F, Duka A, Sun F, Cui J, Manolis A, Gavras H: Mitochondrial genome mutations in hypertensive individuals. $\mathrm{Am} \mathrm{J}$ Hypertens 2004, 17:629-635.

27. Yang Q, Kim SK, Sun F, Cui J, Larson MG, Vasan RS, Levy D, Schwartz $\mathrm{F}$ : Maternal influence on blood pressure suggests involvement of mitochondrial DNA in the pathogenesis of hypertension: the Framingham Heart Study. Hypertension 2007, 25:2067-2073.

28. Basanez G, Zhang J, Chau BN, Maksaev GI, Frolov VA, Brandt TA, Burch J, Hardwick JM, Zimmerberg J: Pro-apoptotic cleavage products of $\mathrm{Bcl}-\mathrm{xL}$ form cytochrome c-conducting pores in pure lipid membranes. J Biol Chem 200I, 276:3 I083-3 I091.

29. Rostovtseva TK, Antonsson B, Suzuki M, Youle RJ, Colombini M, Bezrukov SM: Bid, but not Bax, regulates VDAC channels. J Biol Chem 2004, 279: I3575-I3583.

30. Heiden MG Vander, Li XX, Gottleib E, Hill RB, Thompson CB, Colombini $\mathrm{M}$ : $\mathrm{Bcl}-\mathrm{xL}$ promotes the open configuration of the voltagedependent anion channel and metabolite passage through the outer mitochondrial membrane. J Biol Chem 200I, 276:19414-19419.

31. Lee HC, Wei YH: Oxidative stress, mitochondrial DNA mutation, and apoptosis in aging. Exp Biol Med 2007, 232:592-606.

\section{Pre-publication history}

The pre-publication history for this paper can be accessed here:

http://www.biomedcentral.com/1471-2350/10/114/pre pub 